\title{
Nanoscale
}

Check for updates

Cite this: Nanoscale, 2021, 13, 17125

\section{Dimensionality-driven metal-insulator transition in spin-orbit-coupled $\mathrm{IrO}_{2} \dagger$}

\author{
E. Arias-Egido, (iD a,b M. A. Laguna-Marco, iD *a,b,c C. Piquer, a,b \\ P. Jiménez-Cavero, (iD ${ }^{a, b}$ I. Lucas, ${ }^{a, b}$ L. Morellón, ${ }^{a, b}$ F. Gallego, ${ }^{d}$ A. Rivera-Calzada, ${ }^{d}$ \\ M. Cabero-Piris, e J. Santamaria, ${ }^{d, f, g}$ G. Fabbris, (D) ${ }^{h}$ D. Haskel, (iD h R. Boada (DD i,j and \\ S. Díaz-Morenoj
}

A metal-insulator transition is observed in spin-orbit-coupled $\mathrm{IrO}_{2}$ thin films upon reduction of the film thickness. In the epitaxially grown samples, the critical thickness ( $t \sim 1.5-2.2 \mathrm{~nm}$ ) is found to depend on growth orientation (001), (100) or (110). Interestingly from the applied point of view, the insulating behavior is found even in polycrystalline ultrathin films. By analyzing the experimental electrical response with various theoretical models, we find good fits to the Efros-Shklovskii-VRH and the Arrhenius-type behaviors, which suggests an important role of electron correlations in determining the electrical properties of $\mathrm{IrO}_{2}$. Our magnetic measurements also point to a significant role of magnetic order. Altogether, our results would point to a mixed Slater- and Mott-type of insulator.

Received 29th June 2021 Accepted 15th September 2021 DOI: $10.1039 / \mathrm{d} 1 \mathrm{nr} 04207 \mathrm{f}$ rsc.li/nanoscale

\section{Introduction}

In the last decade, iridium oxides have generated significant excitement due to the observation of unexpected properties such as localized-like transport and magnetism. ${ }^{1-4}$ The ground state of these compounds results from a delicate balance between competing interactions so that it is possible to modify it and tailor their electrical and magnetic behaviors through interface and strain engineering. ${ }^{5-14}$ Upon approaching the two-dimensional limit, the coordination of iridium ions at the interfaces is reduced, typically resulting in a decrease in the

${ }^{a}$ Instituto de Nanociencia y Materiales de Aragón (INMA), CSIC - Universidad de Zaragoza, Zaragoza 50009, Spain. E-mail: anlaguna@unizar.es

${ }^{b}$ Departamento de Física de la Materia Condensada, Universidad de Zaragoza,

Zaragoza 50009, Spain

${ }^{c}$ Instituto de Ciencia de Materiales de Madrid, CSIC, Cantoblanco, 28049 Madrid, Spain

${ }^{d}$ GFMC, Universidad Complutense de Madrid, 28040 Madrid, Spain

${ }^{e}$ ICTS - Centro Nacional de Microscopía Electrónica, Universidad Complutense de Madrid, 28040 Madrid, Spain

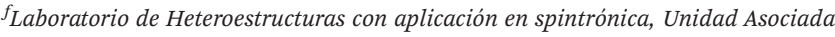
UCM/CSIC, 28049 Madrid, Spain

${ }^{g} G F M C$, Instituto de Magnetismo Aplicado, Universidad Complutense de Madrid, 28040 Madrid, Spain

${ }^{h}$ Advanced Photon Source, Argonne National Laboratory, Argonne, IL 60439, USA ${ }^{i}$ Department of Chemistry Universitat Autonoma de Barcelona 08193 Bellaterra, Barcelona, Spain

${ }^{j}$ Diamond Light Source Ltd Harwell Science and Innovation Campus Didcot, OXfordshire OX11 ODE, UK

$\dagger$ Electronic supplementary information (ESI) available: Structural information of the films and their XANES characterization. See DOI: 10.1039/d1nr04207f electronic bandwidth $W$. Similarly, strain engineering resulting in longer bond lengths or octahedral rotations typically results in a decrease of $W$. Hence, by thickness (dimensionality) control and/or strain engineering, a critical point may be reached where a metal-insulator transition (MIT) is induced.

Benefitting from this tunability, one can not only change the electronic structure and electrical/magnetic behavior but also shed light on the mechanisms determining the electronic and magnetic properties of iridates. Three mechanisms have been proposed to describe the modification of the electronic structure as the result of the reduction of bandwidth, namely, the reduction of $W$ may (i) result in an increase in the effective correlation $(U / W$, where $U$ is the on-site Coulomb repulsion), (ii) make the disorder $(D)$ more effective in causing Anderson (weak) localization (i.e. $D / W$ increases) and (iii) lead to an antiferromagnetic ordering transition resulting in a Slater insulator. In consequence, the origin of the MIT in iridates is still under extensive debate (Mott vs. Anderson vs. Slater) as illustrated by numerous works on the compounds of the Ruddlesden-Popper series, $\mathrm{Sr}_{n+1} \operatorname{Ir}_{n} \mathrm{O}_{3 n+1}(n=1,2, \infty){ }^{1,8,10-13,15-17}$

In $\mathrm{IrO}_{2}, U / W$ and $D / W$ are expected to be smaller than in other iridates, such as the Ruddlesden-Popper series, due to its higher connectivity based on the three-dimensional cornersharing and edge-sharing octahedral network. In fact, there is a wide consensus regarding the metallic and paramagnetic nature of bulk $\mathrm{IrO}_{2}$. On the other hand, theoretical studies suggest that the metallic paramagnetic ground state of $\mathrm{IrO}_{2}$ can be tuned through structural modifications. In this context, Panda et al. ${ }^{18}$ predicted that $\mathrm{IrO}_{2}$ may transform into an antiferromagnetic metal and eventually into an antiferromagnetic 
insulator when $U / W$ increases. Similarly, Kahk et al. ${ }^{19}$ predicted that epitaxial strain may strongly affect the electronic structure through its different effects on orbitals within $\left(\mathrm{d}_{x^{2}-y^{2}}\right)$ and perpendicular $\left(\mathrm{d}_{x z}, \mathrm{~d}_{y z}\right)$ to the edge sharing planes. With a similar view, Ming et al. ${ }^{20}$ focused on the evolution of the electronic structure as a function of the $\mathrm{IrO}_{2}$ layer thickness, $m$, in $\left(\mathrm{IrO}_{2}\right)_{m}\left(\mathrm{TiO}_{2}\right)_{10}$ heterostructures, and predicted by the densityfunctional theory that the heterostructures exhibit a thicknesscontrolled MIT. According to their work, the subtle interplay between electron correlation and spin-orbit coupling (SOC) leads to an almost pure $J_{\text {eff }}=1 / 2$ spin-orbit insulating state at the level of the atomically-thin monolayer. These predicted emerging insulating and magnetic states are still awaiting experimental confirmation.

Here, we investigate the electrical and magnetic properties of (001)-, (100)-, and (110)-epitaxially grown thin $\mathrm{IrO}_{2}$ films of different thicknesses to directly address the effect of dimensionality and strain. We find that an MIT occurs at a film thickness of $\sim 1.5 \mathrm{~nm}$, i.e. $\sim 4$ unit cells (u.c.). Moreover, our results point to the possibility of achieving an MIT also in polycrystalline (110)-textured films. The mechanisms responsible for the MIT have been studied by fitting the resistivity curves to several models. In addition, our magnetization and XMCD data suggest the appearance of magnetic ordering in $\mathrm{IrO}_{2}$ through thickness reduction as proposed by theoretical predictions. ${ }^{18,20}$ Our study highlights ultra-thin $\mathrm{IrO}_{2}$ as a novel building block for engineering the interplay of spin-orbit coupling, electrical resistivity and magnetism.

\section{Experimental}

Epitaxial $\mathrm{IrO}_{2}$ films of thicknesses $5 \mathrm{~nm}$ and $100 \mathrm{~nm}$ were deposited on $\mathrm{TiO}_{2}$ single-crystal substrates with (100), (001) and (110) orientation by pulsed laser deposition (PLD) using a KrF excimer laser with $248 \mathrm{~nm}$ wavelength, $3 \mathrm{~Hz}$ repetition rate, and $1.5 \times 10^{9} \mathrm{~W} \mathrm{~cm}^{-2}$ irradiance in an ultrahigh-vacuum chamber using a deposition temperature of $380{ }^{\circ} \mathrm{C}$. Epitaxial films thinner than $5 \mathrm{~nm}$ were deposited on $\mathrm{TiO}_{2}$ substrates with (100), (001) and (110) orientation by high pressure sputtering in pure oxygen atmosphere, a technique which has shown the growth of complex oxides with good epitaxial properties. ${ }^{21}$ \$ The substrate to target distance was fixed at $1.5 \mathrm{~cm}$, so that the plasma is tangent to the target surface. The sputtering power was set to $110 \mathrm{~W}$ and the oxygen pressure to 3.2 mbar. The substrate temperature was set to RT. Interestingly, the crystal quality of the samples gets worse if the substrate temperature is set above $400{ }^{\circ} \mathrm{C}$. It is worth noting the importance and novelty of this result since the growth of other TMOs carried out by means of this technique typically requires growing temperatures above $750{ }^{\circ} \mathrm{C} .{ }^{22,23}$ Different deposition times were used to obtain different thick-

$\$$ Samples with $t \sim 5 \mathrm{~nm}$ were also grown by high pressure sputtering and used to confirm that the resistivity behaviour does not depend on the deposition method but they are not shown here. nesses. In addition, polycrystalline (110)-textured films, labeled as (110)-text, were grown in a conventional reactive magnetron sputtering equipment from an iridium target and using an $\mathrm{O}_{2} / \mathrm{Ar}$ mixture gas that is $\sim 13.3 \% \mathrm{O}_{2}$-rich. The base pressure provided by the vacuum system was in the $10^{-7} \mathrm{mbar}$ range and the working pressure was $\sim 5 \times 10^{-3}$ mbar. The DC power used to grow the samples was about $8 \mathrm{~W}$. The polycrystalline samples were grown on $\mathrm{Si}(100)$ substrates at RT and subsequent thermal annealing was performed in air at $650{ }^{\circ} \mathrm{C}$ for 6 hours.

$\mathrm{X}$-ray reflectivity (XRR) and X-ray diffraction measurements (XRD) were performed by using Bruker D8 and Rigaku D/max2500 diffractometers, respectively, by using the $\mathrm{K}_{\alpha}$ radiation line of copper. Field-emission scanning electron microscopy (FE-SEM) images were recorded on the surface of representative samples to investigate the morphology of the films. Image recording was performed at $15 \mathrm{kV}$ by using a Carl Zeiss MERLIN microscope. The electrical resistivity was measured using the four points van der Pauw method ${ }^{24}$ by means of a Quantum Design PPMS 9 T from 300 to $10 \mathrm{~K}$ with no applied magnetic field and with a small electric current $(0.1 \mathrm{~mA})$. Magnetization measurements were carried out by using a commercial SQUID magnetometer from Quantum Design. Magnetization hysteresis loops were achieved up to $50 \mathrm{kOe}$ at RT and 5 K. Magnetization versus temperature data were collected from 5 to $350 \mathrm{~K}$ with a heating rate of $5 \mathrm{~K} \mathrm{~min}^{-1}$ at 1000 Oe.

High-energy resolution fluorescence detected X-ray absorption spectroscopy (HERFD-XANES) measurements were carried out at RT by using a beamline I20-Scanning at Diamond Light Source. ${ }^{25-27}$ Further details can be found elsewhere. ${ }^{27,28}$ X-ray magnetic circular dichroism (XMCD) measurements were carried out at beamline 4-ID-D of the Advanced Photon Source, Argonne National Laboratory. Measurements were done in a cryomagnet with the sample cooled with ${ }^{4} \mathrm{He}$ vapor. A $500 \mu \mathrm{m}$ thick diamond phase plate was used to generate circularly polarized X-rays and XMCD measurements were carried out in helicity-switching mode. Measurements were done in fluorescence mode using a grazing incidence geometry and an energy dispersive 4-element Si drift diode detector placed at 90 degrees relative to the incident beam direction. Data were collected at $10 \mathrm{~K}$ with the magnetic fields along and opposite the $\mathrm{X}$-ray propagation direction to remove any artifacts of nonmagnetic origin.

\section{Results and discussion}

$\mathrm{IrO}_{2}$ films of different thicknesses from $100 \mathrm{~nm}$ down to $1.5 \mathrm{~nm}$ were obtained (Table 1 ). In the case of the thicker films $(t>3 \mathrm{~nm})$, the thickness was calculated from XRR data (see the ESI $\dagger)$. For the thinner samples, $(t \leq 3 \mathrm{~nm}) t$ was calculated from XRD data (Fig. 1) by using the Scherrer formula.

The panels (a), (b) and (c) of Fig. 1 show the HR-XRD data of the epitaxially grown $\mathrm{IrO}_{2}$ samples. Only Bragg peaks corresponding to the substrate orientation were observed in each 
Table 1 Structural details of the $\mathrm{IrO}_{2}$ films: growth orientation, thickness and lattice parameters along with their relative differences with the bulk values

\begin{tabular}{lllll}
\hline Orientation & $t(\mathrm{~nm})$ & $a\left(\varepsilon_{\mathrm{a}}\right)$ & $b\left(\varepsilon_{\mathrm{b}}\right)$ & $c\left(\varepsilon_{\mathrm{c}}\right)$ \\
\hline$(001)$ & 96.0 & - & - & - \\
$(001)$ & 5.7 & $4.59(+2.0 \%)$ & $4.59(+2.0 \%)$ & $3.11(-1.6 \%)$ \\
$(001)$ & 2.5 & - & - & - \\
$(001)$ & 2.2 & - & - & - \\
$(001)$ & 1.5 & - & - & - \\
& & & & \\
$(100)$ & 89.0 & $4.46(-0.9 \%)$ & $4.59(+2.0 \%)$ & $3.13(-0.9 \%)$ \\
$(100)$ & 5.1 & $4.48(-0.4 \%)$ & $4.59(+2.0 \%)$ & $3.10(-1.9 \%)$ \\
$(100)$ & 1.7 & - & - & - \\
$(100)$ & 1.5 & - & - & - \\
$(110)$ & 92.2 & - & - & - \\
$(110)$ & 5.3 & - & - & - \\
$(110)$ & 2.7 & - & - & - \\
$(110)$ & 2.2 & - & - & - \\
& & & - & - \\
$(110)$-text & 106.5 & $4.49(-0.2 \%)$ & $4.49(-0.2 \%)$ & $3.13(-0.9 \%)$ \\
$(110)$-text & 6.3 & - & - & - \\
$(110)$-text & 3.3 & - & - & -
\end{tabular}

${ }^{a}$ Estimated from deposition time. sample as expected from their epitaxial growth. The peak position is slightly dependent on the layer thickness indicating modifications of the out of plane lattice parameter resulting from epitaxial strain. The lattice parameters for the (001) and (100)-epitaxial films were calculated from the reciprocal space maps collected on selected $100 \mathrm{~nm}$ - and $5 \mathrm{~nm}$-thick samples (see Fig. S4 in the ESI $\dagger$ ). The values are summarized in Table 1. As rutile $\mathrm{TiO}_{2}$ substrates with nominal lattice parameters $a=b=4.59 \AA$ and $c=2.96 \AA$ were employed, the $\mathrm{IrO}_{2}$ films present different degrees of tensile/compressive strain depending on substrate orientation. Thus, the $t \sim 5 \mathrm{~nm}$-thick (001)-epitaxial sample presents a large tensile in-plane strain that is not fully compensated by a decrease of the out-of plane $c$ direction, causing a significant modification of the unit cell volume $\left(\varepsilon_{\mathrm{a}}=\varepsilon_{\mathrm{b}}=2.0 \%, \varepsilon_{\mathrm{c}}=-1.6 \%\right.$ and $\left.\Delta V / V_{0}=2.4 \%\right)$. In contrast, the (110)-textured sample presents almost unstrained $a$ and $b$ lattice parameters $\left(\varepsilon_{\mathrm{a}}=\varepsilon_{\mathrm{b}}=-0.2 \%\right)$ and a small compression along the $c$ direction $\left(\varepsilon_{\mathrm{c}}=-0.9 \%\right)$ resulting in a volume decrease $\Delta V / V_{0}=-1.4 \%$. On the other hand, growing the films with (100) orientation barely modifies the total volume $\left(\left|\Delta V / V_{0}\right|<0.4 \%\right)$ but results in remarkably dissimilar $a$
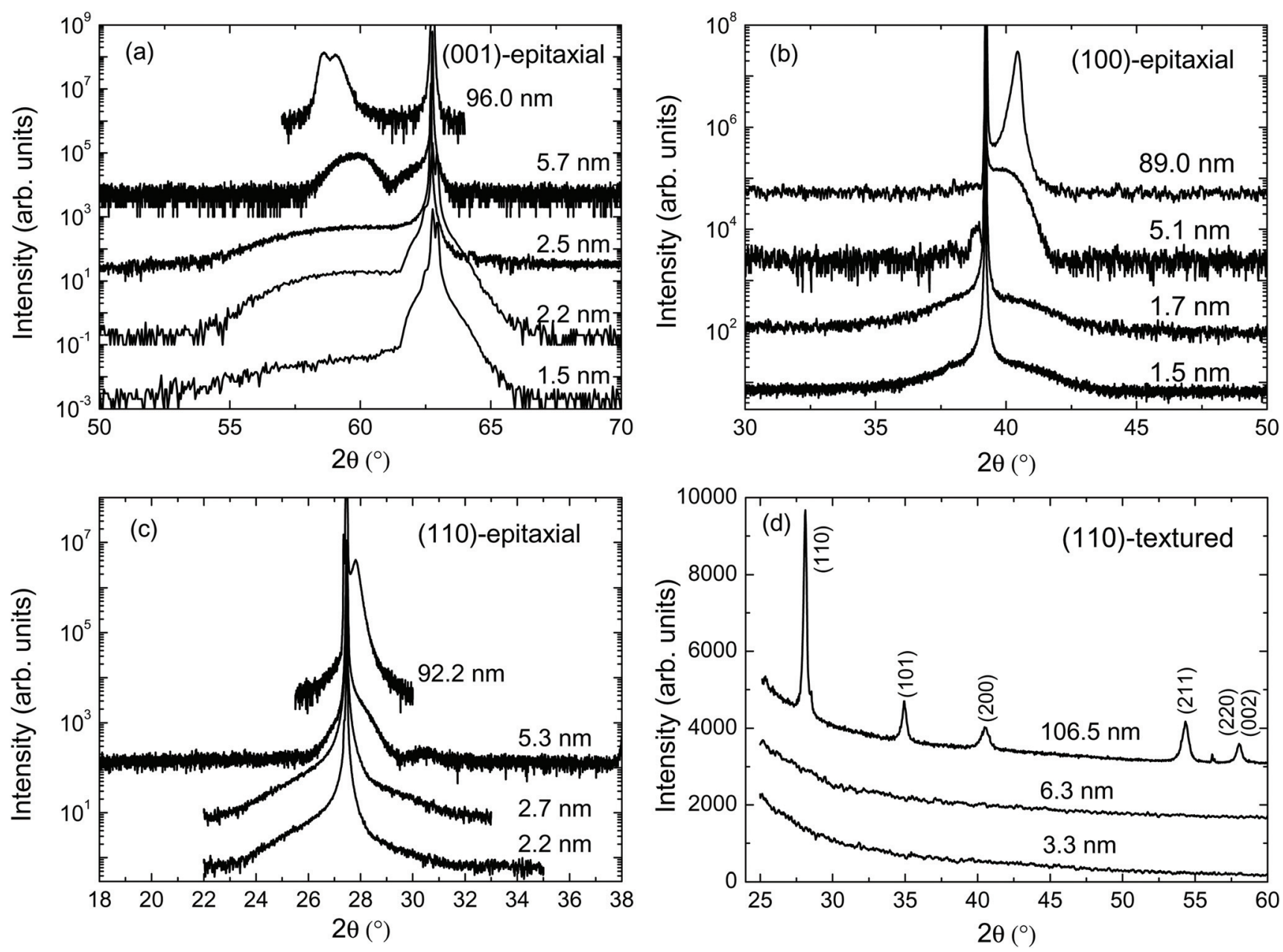

Fig. 1 XRD measurements of the (a) (001)-epitaxial, (b) (100)-epitaxial, (c) (110)-epitaxial and (d) (110)-textured films with different thickness. 
and $b$ parameters. It is to be noted here that the $a, b$ and $c$ unit cell axes are not oriented along the octahedral axis in the rutile structure.

Further structural characterization of the films is exemplified in Fig. 2. A flat surface morphology is confirmed for ultrathin films by atomic force microscopy topography. It can be seen that both sample and substrate present similar roughness with maximum $Z$ heights below $0.6 \mathrm{~nm}$ and $\mathrm{rms} \sim 0.2 \mathrm{~nm}$, thus showing the continuity of the film. A flat interfacial plane is also observed in the cross-sectional STEM images. Crystal defects are not substantially detected. Representative rocking curves, further showing the good structural quality of the films have been included in the ESI. $\dagger$

The (110)-textured polycrystalline film with $t \sim 100 \mathrm{~nm}$ displays the typical peaks of rutile-IrO $\mathrm{Ir}_{2}$, with the relative intensity of the (110)-peak clearly enhanced (Fig. 1(d)). For smaller thicknesses, no diffraction peaks can be discerned due to the small amount of material present in the thinnest samples. Even when some loss of crystallinity cannot be ruled out, the well-defined features in the XAS spectra (see Fig. S3 in the $\mathrm{ESI} \dagger$ ) confirm their crystalline (non-amorphous) character.

The HERFD-XANES spectra were recorded at the Ir $\mathrm{L}_{2,3}$ edges to get a hint on the possible changes in both the electronic structure and SOC. (The branching ratio, i.e. the ratio of the integrated white line intensity recorded at the $\operatorname{Ir} L_{2,3}$ absorption edges, $\mathrm{BR}=I_{\mathrm{L} 3} / I_{\mathrm{L} 2}$, is related to the angular part of the spin-orbit interaction $\langle\mathbf{L} \cdot S\rangle$, see the ESI $\dagger$ for details). Very similar spectral profiles can be observed irrespective of the degree of crystallinity, thickness and strain; therefore, large and mostly constant SOC values are inferred in all the cases, as shown in the representative case displayed in Fig. 3. Nevertheless, a slightly increasing branching ratio is found as
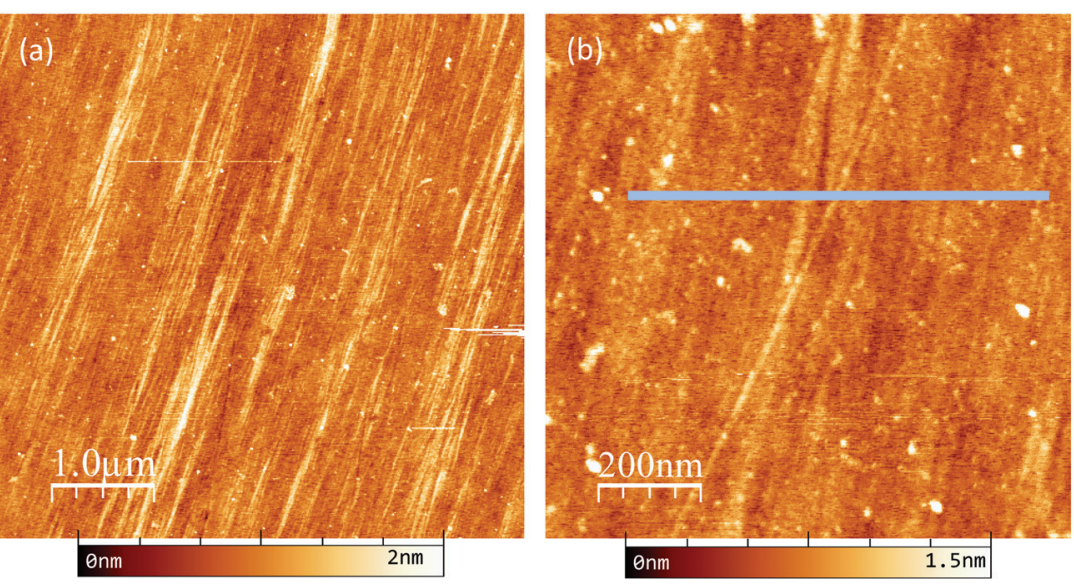

(c)

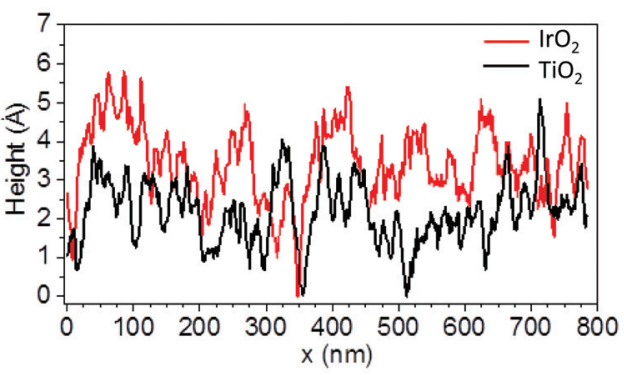

rms:0.16nm

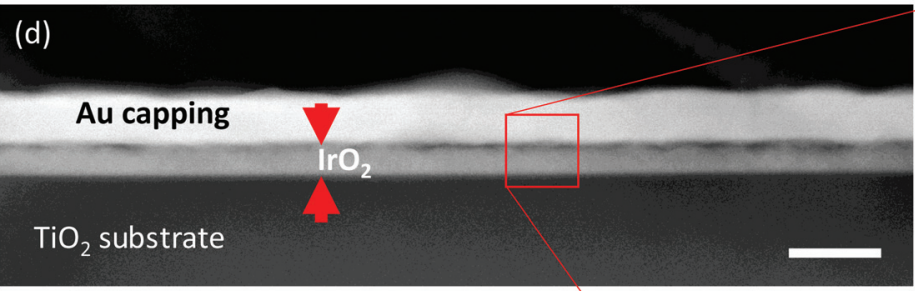

(e)

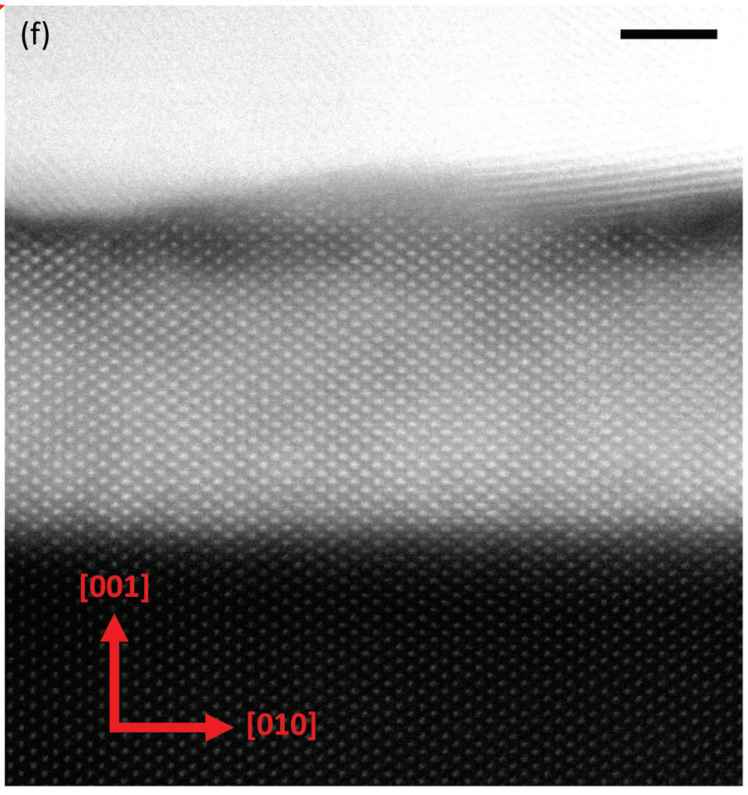

Fig. 2 (a) and (b) RT Atomic force microscopy (AFM) image of a $2 \mathrm{~nm}$ (001) $\mathrm{IrO}_{2}$ film.(c) Profile along the line shown in (b) compared to the profile measured on a $\mathrm{TiO}_{2}$ substrate. The root mean square roughness is $0.16 \mathrm{~nm}$. (d) -(f) STEM high angle annular dark field images of a $\sim 5 \mathrm{~nm}$ IrO $\mathrm{O}_{2}$ thin film on the $\mathrm{TiO}_{2}$ [001] susbtrate. (d) and (e) low magnification images and (f) high resolution image of the framed area in (d) show the epitaxial IrO $\mathrm{I}_{2} /$ $\mathrm{TiO}_{2}$ interface. Red arrows indicate the zone axis of the $\mathrm{TiO}_{2}$ [001] substrate. Scale bars are $20 \mathrm{~nm}, 5 \mathrm{~nm}$ and $2 \mathrm{~nm}$, respectively. 


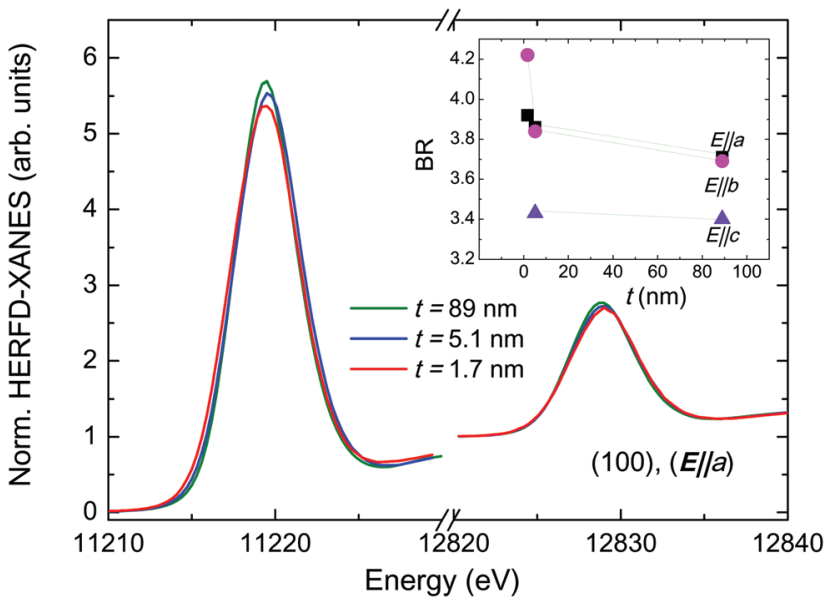

Fig. 3 Comparison of the normalized Ir $\mathrm{L}_{2,3}$-edges HERFD-XANES spectra recorded on samples with different thicknesses in (100)-epitaxial samples. The inset plots the evolution of the branching ratio with thickness. the thickness is reduced. Although the differences between $100 \mathrm{~nm}$ and $1.5 \mathrm{~nm}$ thick samples are in general smaller than $10 \%$, the trend is robust. In contrast, no clear trend can be discerned as the strain is modified by the growth on substrates with different crystalline orientations (See the ESI $\dagger$ for a detailed discussion).

All the thick samples (i.e. $t>3 \mathrm{~nm}$ ) present the typical metallic behavior of $\mathrm{IrO}_{2}$ characterized by a monotonically slightly increasing electrical resistivity, $\mathrm{d} \rho / \mathrm{d} T>0$, over the whole temperature range (Fig. 4). For the thickest samples, $t \sim$ $90 \mathrm{~nm}$, electrical resistivity in the $30-55 \mu \Omega \mathrm{cm}$ range is obtained at the lowest temperature, $T=10 \mathrm{~K}$, with residual resistivity ratios, $\mathrm{RRR}=R(300 \mathrm{~K}) / R(10 \mathrm{~K})$, in the 1.6-2.0 range (detailed table in the ESI $\dagger$ ). These values are in good agreement with other previously reported values for epitaxial films. ${ }^{29-31}$ The low temperature-dependent contribution $\rho(T)$ fits well to a $T^{2}$-dependence indicating that the electron-electron scattering dominates the temperature dependent part of the electrical resistivity, i.e., a typical Fermi liquid behavior.
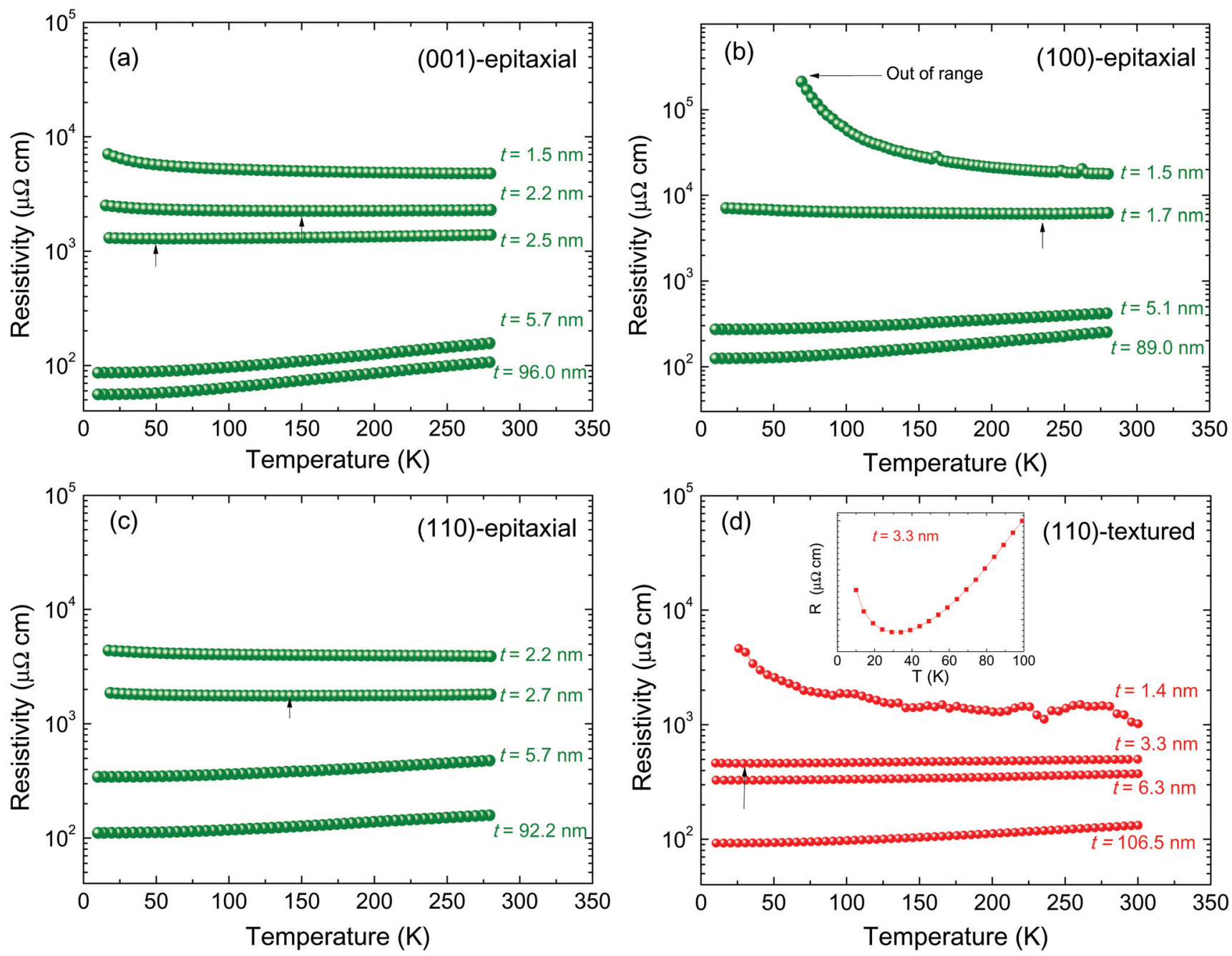

Fig. $4 \rho(T)$ curves for the (a) (001)-epitaxial, (b) (100)-epitaxial, (c) (110)-epitaxial and (d) (110)-textured samples with different thicknesses. Vertical arrows mark the position of upturns. 
As $t$ decreases, not only the residual resistivity due to defects, impurities, size effects, and grain boundary scattering increases but also the thermal dependence varies. For the 2-3 nm-thick films, a slope change from $\mathrm{d} \rho / \mathrm{d} T>0$ at high temperature to $\mathrm{d} \rho / \mathrm{d} T<0$ at low temperature is observed at $T_{\text {min }}$, marked with arrows in Fig. 4 (zoomed images in the ESI $\dagger$ ). This kind of low temperature upturns in resistivity is often observed when a system approaches an MIT from the metallic side. ${ }^{13,32-34}$ Furthermore, $T_{\min }$ is located at increasingly higher temperatures as the thickness is reduced. This behavior is related to the progressive localization of the system with thickness reduction, which, in turn, makes the bands closer to the Fermi level progressively depopulated for a given temperature. In this respect, a similar behavior observed in $\mathrm{SrIrO}_{3}$ has been proposed to be related to the semimetallic electronic structure where the hole bands lying just below the Fermi level will be progressively depopulated with decreasing temperature. ${ }^{8}$ For the thinnest samples $(t=1.5 \mathrm{~nm}$ for the (001) and (001) samples and $t=2.2 \mathrm{~nm}$ for the (110) sample), $\mathrm{d} \rho / \mathrm{d} T<0$ is observed in the whole temperature range. Therefore, it is concluded that the $\mathrm{IrO}_{2}$ crystalline films undergo a thickness-driven MIT within the 1.5-2.5 $\mathrm{nm}$ range. Such layer thicknesses correspond to 3-5 unit cells, being quite in agreement with the theoretical predictions. ${ }^{18,20}$ The comparison between the different panels in Fig. 4 indicates that thickness reduction is more effective in inducing an MIT in the case of (100)-oriented samples and less effective for (001) orientation. Interestingly from the applied point of view, the thinnest polycrystalline (110)-textured film also shows a $\mathrm{d} \rho / \mathrm{d} T<0$ behavior over the whole temperature range.

While recent works on $\mathrm{IrO}_{2} / \mathrm{TiO}_{2}$ superlattices ${ }^{35}$ could not find this MIT, it has to be noted that the superlattices of ref. 35 are distinctly different from $\mathrm{IrO}_{2}$ single films, since the former may be affected by interlayer coupling by ultrathin (2 atomic planes) $\mathrm{TiO}_{2}$ spacers. In particular, the superlattices are probably affected by additional hopping channels between the $\mathrm{IrO}_{2}$ layers, as observed in $\left(\mathrm{SrIrO}_{3}\right) /\left(\mathrm{SrTiO}_{3}\right)_{m}$ superlattices. ${ }^{36}$ By studying single $\mathrm{IrO}_{2}$ layers of different thicknesses, the effect of dimensionality on $\mathrm{IrO}_{2}$ is directly addressed.

Fig. 5(a) shows the sheet resistance, $R_{\mathrm{s}}$, as a function of temperature for representative samples $\left(R_{\mathrm{S}}=R \pi / \ln (2)\right.$, with $R$ being the electrical resistance). It can be seen that $R_{\mathrm{S}}$ continuously increases as the film thickness is reduced. In addition, the change in the electrical behavior occurs when $R_{\mathrm{S}}$ crosses the $25 \mathrm{k} \Omega$ value, i.e. the so-called Mott-Ioffe-Regel limit. In very thin films $(\sim 2 \mathrm{D}), k_{\mathrm{F}} l=\left(\mathrm{h} / \mathrm{e}^{2}\right) / R_{\mathrm{S}} \approx 25 \mathrm{k} \Omega / R_{\mathrm{S}}$, with $l$ being the mean free path and $k_{\mathrm{F}}$ the Fermi wave-vector. $R_{\mathrm{S}}=25 \mathrm{k} \Omega$ corresponds to the limit where $k_{\mathrm{F}} l=1$. The above criterion defines a crossover between weak and strong localization. ${ }^{37}$

Trying to shed some light on the mechanisms underlying the MIT, the most resistive curves were fitted to several models, see Fig. 5(b) and (c). The resistivity of the (001)-epitaxial film with $t=1.5 \mathrm{~nm}$ has a sheet resistance very close to $\mathrm{h} / \mathrm{e}^{2}$ at RT and an insulating behavior is found at all temperatures. For this strongly localized regime, a variable range hopping
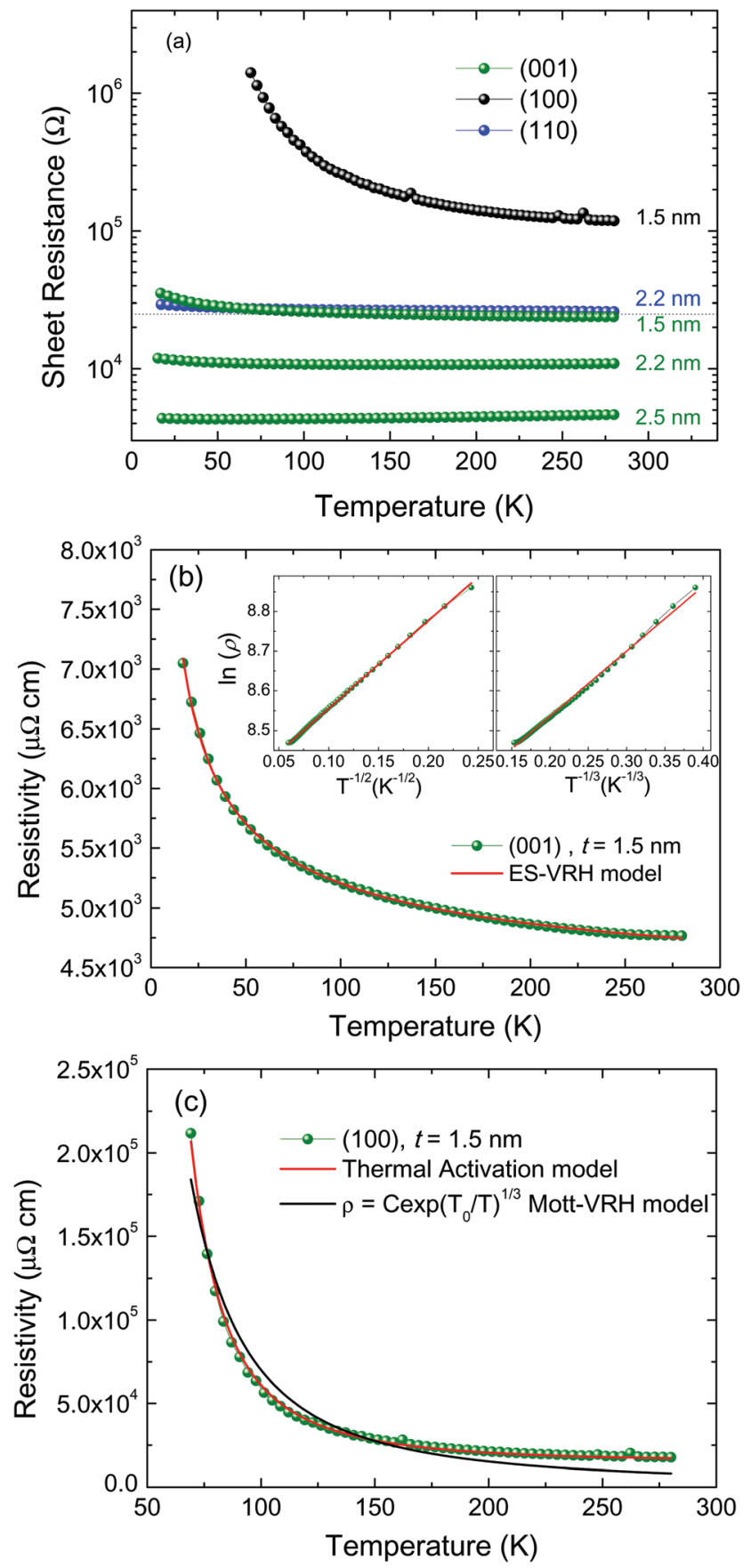

Fig. 5 (a) Sheet resistance calculated for the thinnest (001)- and (100)epitaxial films. (b) $\rho(T)$ curve for the $t=1.5 \mathrm{~nm}$ (001)-epitaxial sample fitted to a ES-VRH model. Inset on the left (right) shows the fit to the ES-VRH model with $n=1 / 2$ (disorder-driven localization 2D Mott-VRH model, $n=$ 1/3). (c) $\rho(\mathrm{T})$ curve for the $t=1.5 \mathrm{~nm}$ (100)-epitaxial sample fitted to a thermal activation model (red) and a 2D Mott-VRH model (black).

(VRH) type of conduction is expected. Electrons hop between the localized states and the resistivity is given by: ${ }^{38,39}$

$$
\rho=C \exp \left(T_{0} / T\right)^{n}
$$

where $C$ is a constant and $T_{0}$ is the characteristic temperature of the compound, which depends on the density of the loca- 
lized states and the spread of their wave functions. The fitting shown in Fig. 5(b) for the (001)-epitaxial sample with $t=$ $1.5 \mathrm{~nm}$ yields an exponent $n=0.46$, being in good agreement with the Efros-Shklovskii VRH (ES-VRH) model $(n=0.5)$. Such a model assumes a Coulomb gap opening in the density of states near the Fermi level brought in by interactions between localized electrons, i.e., a Mott insulator. ${ }^{40}$ As for the sample with the highest electrical resistivity, i.e. the (100)-epitaxial film with $t=1.5 \mathrm{~nm}$, as shown in Fig. 5(c), its behavior is best described by an Arrhenius type behavior given by:

$$
\rho=C \exp \left(E_{\mathrm{g}} / 2 K_{\mathrm{B}} T\right)
$$

where $C$ is a constant, $E_{\mathrm{g}}$ is the energy gap and $K_{\mathrm{B}}$ is the Boltzmann constant. This fit yields an energy gap $E_{\mathrm{g}}$ of $\sim 30 \mathrm{meV}$.

Therefore, we find that the thickness reduction of $\mathrm{IrO}_{2}$ films yields an MIT. Best fits are obtained in the case of the thinnest samples to either an ES-VRH or an Arrhenius model. Both the hopping (ES-VRH) and thermal activation mechanisms indicate a gap opening that may be associated with an enhanced role of correlations. Nevertheless, several mechanisms could coexist and, for example, an additional contribution of disorder should not be ruled out at this point. In fact, the low temperature region of resistivity curves showing an upturn has been commonly accounted for in terms of weak (Anderson) localization, a disorder-driven effect due to the quantum interference of the conducting charge carriers at defect sites. In the same way, a dissimilar presence of structural defects combined with the ultrathin thickness could also be contributing to the differences in resistivity observed in the work by Kawasaki et al. ${ }^{41}$ Thus, although the TEM characterization of our samples is in disagreement with the presence of a substantial amount of defects and a major role of disorder, an additional contribution of disorder should not be ruled out. Similarly, the role of SOC and spin fluctuations should be explored. In this regard, it seems unlikely that the little enhancement of the SOC inferred from our data alone may drive the MIT. On the other hand, as a result of the enhanced localization in the thinnest samples ( $W$ reduction), the effective SOC increases and the role of the SOC may become more relevant. The presence of the SOC could, for instance, reduce the value of $U$ necessary for the MIT. Further work is required to fully describe the nature of this thickness-driven MIT and the role of the different interactions.

While no MIT has been reported for single $\mathrm{IrO}_{2}$ thin films to date, it may be illustrative at this point to compare with previous works on $\mathrm{SrIrO}_{3}$ thin films of variable thicknesses. Thus, our experimental findings are in agreement with those reported by Groenendijk et al., ${ }^{8}$ who point out the major role of correlations.

In contrast to the thickness dependence, no significant modification of $\rho(T)$ can be attributed to a strain-driven effect. As observed in Fig. 6, samples with large differences in their lattice parameters display very similar resistivity curves. For instance, the $t \sim 5 \mathrm{~nm}$-thick (001)-epitaxial sample (with $\varepsilon_{\mathrm{a}}=$ $2.0 \%, \varepsilon_{\mathrm{b}}=2.0 \%$ and $\left.\varepsilon_{\mathrm{c}}=-1.6 \%\right)$ presents a very similar resistivity to that of the (110)-textured sample (with $\varepsilon_{\mathrm{a}}=-0.2 \%, \varepsilon_{\mathrm{b}}=$

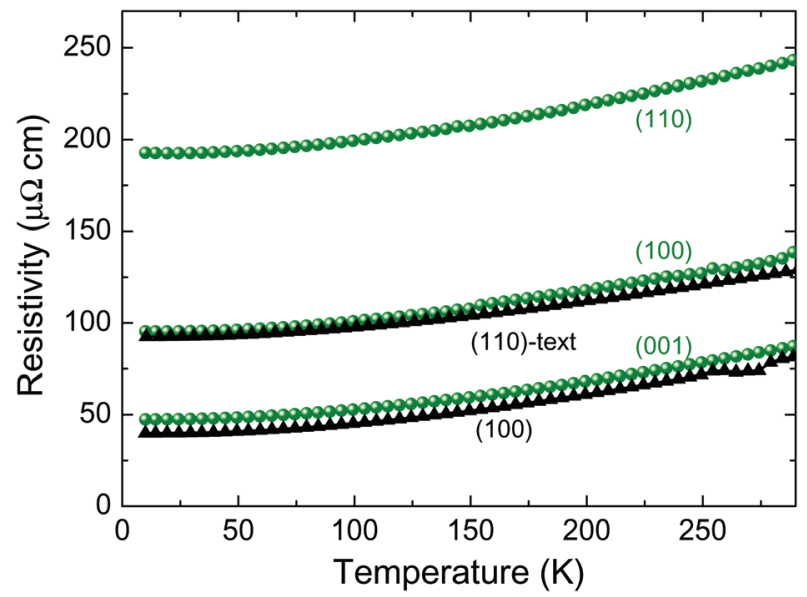

Fig. 6 Comparison of the representative $\rho(T)$ curves measured in samples with different substrate-driven strains, $t \approx 5 \mathrm{~nm}$ (green circles) and $t \approx 100 \mathrm{~nm}$ (black triangles).

$-0.2 \%$ and $\left.\varepsilon_{\mathrm{c}}=-0.9 \%\right)$ This behavior is noticeably different from that observed in $\mathrm{SrIrO}_{3}$, where a $35 \mathrm{~nm}$-thick film deposited on $\mathrm{NdGaO}_{3}$ (with $\varepsilon_{\mathrm{a}}=2.5 \%$ and $\varepsilon_{\mathrm{c}}=-2.7 \%$ ) results in $\mathrm{d} \rho / \mathrm{d} T<0$ over the whole temperature range. ${ }^{11,13}$ Such a different response to the substrate-driven strain could be related to the structural differences of these iridates; while the $\mathrm{IrO}_{6}$ octahedra in $\mathrm{IrO}_{2}$ share corners and edges, in $\mathrm{SrIrO}_{3}$ they are only corner-sharing. This results in an initially broader bandwidth as well as in a more rigid structure in the case of $\mathrm{IrO}_{2}$. Consequently, although a similar substrate-driven strain is achieved in both $\mathrm{SrIrO}_{3}$ and $\mathrm{IrO}_{2}$ cases, it seems not to be enough to significantly reduce $W$ in $\mathrm{IrO}_{2}$.

It is be noted, however, that a strain-driven effect still could become important in combination with ultrathin films. In this sense, Kawasaki et $a l .{ }^{41}$ report only a slight upturn at low temperatures for ultrathin $\mathrm{IrO}_{2}$ film. We propose that these differences in the transport behaviour can be ascribed to different lattices matching with the substrate and so to different lattice parameters and distortions.

No hint of magnetic order is observed in the $M(H)$ measurements regardless of the thickness, growth orientation or strain (not shown). On the other hand, the temperature-dependent magnetization curves suggest that magnetic order may appear for thin enough films. In particular, all the samples but the most insulating one show a roughly paramagnetic behavior, typically expected for $\mathrm{IrO}_{2}$, as illustrated by the curve recorded on the 1.5 nm-thick (001)-epitaxial film (Fig. 7(a)). However, a weak ferromagnetic-like transition with $T_{\mathrm{C}} \sim 125 \mathrm{~K}$ is observed in the $1.5 \mathrm{~nm}$-thick (100)-epitaxial film superimposed to the paramagnetic response (Fig. 7(b)). Zero-field-cooled (ZFC) and field-cooled (FC) curves split at a temperature slightly below $T_{\mathrm{C}}$. This fact might indicate that weak ferromagnetism arises from a canted antiferromagnetic order, as occurs in $\mathrm{Sr}_{2} \mathrm{IrO}_{4}{ }^{42}$ or Sn-doped $\mathrm{SrIrO}_{3},{ }^{15,17}$ and is also in agreement with the theoretical predictions on $\mathrm{IrO}_{2} \cdot{ }^{18,20}$ It should be noted here that macroscopic magnetic data can hardly unambiguously 

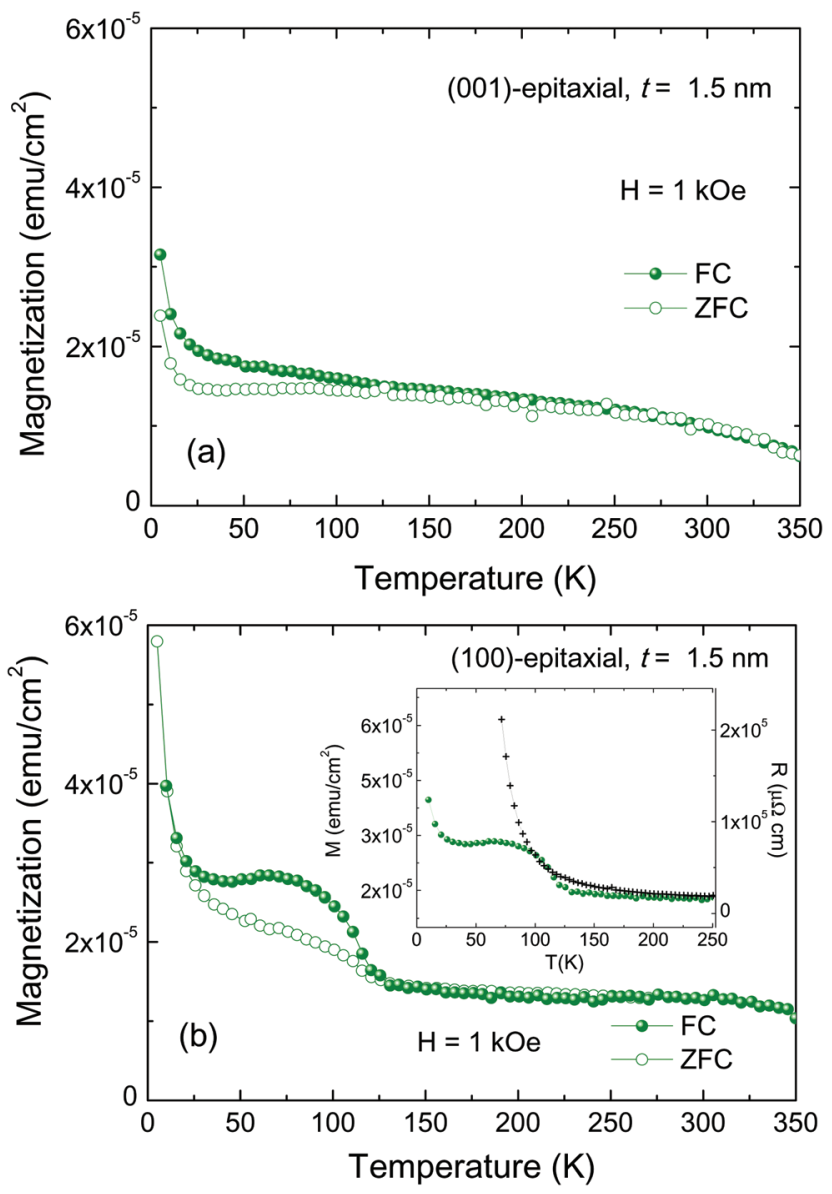

Fig. 7 (a) FC/ZFC M(T) curves measured on the $t=1.5 \mathrm{~nm}$ (001)-epitaxial $\mathrm{IrO}_{2}$ film. These curves correspond to $\mathrm{H}$ in-plane, but no hint of magnetic order is observed with $\mathrm{H}$ out-of-plane. (b) FC/ZFC $M(T)$ curves measured with $\mathrm{H}$ applied in-plane on the $t=1.5 \mathrm{~nm}(100)$-epitaxial $\mathrm{IrO}_{2}$ film. The inset of panel (b) shows a direct comparison to the thermal dependence of the resistivity.

confirm or discard a change in the magnetic behavior of such thin films. In particular, the maximum values $\left(\sim 1 \times 10^{-5} \mathrm{emu}\right.$ $\mathrm{cm}^{-2}$ ) expected for these films are not only very small but similar to the typical uncertainties associated with the slightly imperfect correction of the background contribution. Therefore, slight differences in the magnetization cannot be undoubtedly associated with the magnetic behavior of the $\mathrm{IrO}_{2}$ layer. Despite that, it can be observed that the magnetic transition appears approximately in the same temperature range at which electrical resistivity shows a drastic increase (see Fig. 4(b)). This would support that it is indeed associated with the $\mathrm{IrO}_{2}$ layer and a related origin for both magnetic and electric transition. In addition, it is to be noted that the magnetic transition is observed only for the (100)-oriented $1.5 \mathrm{~nm}$-thick film, which is far more resistive than the (001)- and (110)oriented samples. Altogether this suggests a relationship between magnetic and electrical behaviors in such a way that the onset of magnetic ordering would make the sample more resistive. However, the role of magnetic interactions in driving gap formation or gap enhancement has remained a matter of debate in different iridates (see for example ${ }^{43}$ ) and further work is needed to clarify this point.

Contrary to the macroscopic magnetization data, the XMCD data are unambiguously related to the $\mathrm{IrO}_{2}$ layer and allow us to discard any spurious origin. The XMCD spectrum recorded

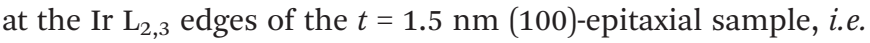
the sample exhibiting the largest electrical resistivity and hints of magnetic ordering in the $M(T)$ curves at low temperatures, is shown in Fig. 8. A small XMCD signal $(0.4 \%)$ can be inferred at the $\mathrm{L}_{3}$ edge. The presence of an XMCD signal at the $\operatorname{Ir} \mathrm{L}_{2}$ edge is ambiguous. If any, its size is much smaller than that at the $\mathrm{L}_{3}$ edge. The position, shape and sign of the small signal observed agree well with previous XMCD measurements carried out in other $\mathrm{Ir}^{4+}$-based compounds, such as in $\mathrm{Sr}_{2} \mathrm{IrO}_{4},{ }^{43} \mathrm{Sr}_{2} \mathrm{TiIrO}_{6}{ }^{44}$ or $\mathrm{BaIrO}_{3}{ }^{45}$ In addition, the XMCD spectrum of powdered $\mathrm{IrO}_{2}$ presents a much smaller intensity (Fig. 8(b)). These comparisons point out that the small XMCD signal may indeed be associated with the magnetic ordering of the $\operatorname{Ir}^{4+}$ cations or, at least, with an enhanced paramagnetic
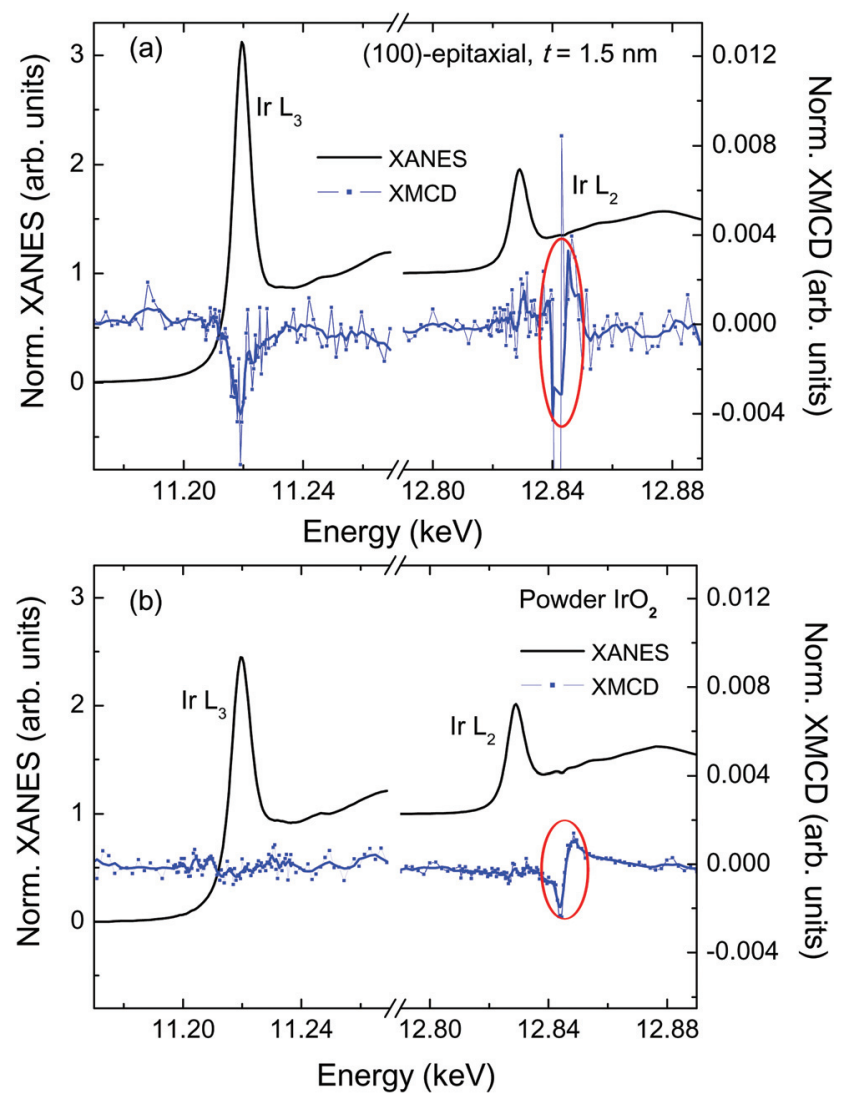

Fig. 8 (a) Normalized XANES and XMCD spectra recorded in the fluorescence mode at the $\operatorname{Ir} L_{2,3}$ edges at $T=10 \mathrm{~K}$ and $H= \pm 50 \mathrm{kOe}$ on the $t$ $=1.5 \mathrm{~nm}$ (100)-epitaxial sample. (b) Normalized XANES and XMCD spectra measured in the transmission mode at the $\operatorname{Ir} L_{2,3}$ edges at $T=$ $10 \mathrm{~K}$ and $\mathrm{H}= \pm 35 \mathrm{kOe}$ on commercial powdered $\mathrm{IrO}_{2}$. The lower intensity of the XAS white line is due to a $4 \%$ of metal Ir phase. Solid blue lines indicate a smoothing of the experimental data (in solid symbols). Red circles indicate a spurious artifact due to a monochromator glitch at that energy. 
signal of this film. Therefore, although further work is needed to verify the presence of magnetic order, these results are the first experimental evidence supporting that magnetic ordering can be achieved in $\mathrm{IrO}_{2}$ through structural modifications (thickness reduction) as proposed by theoretical predictions. ${ }^{18,20}$ In addition, the fact that the magnetic transition appears in the same temperature range where the electrical resistivity drastically increases might indicate an important role of magnetic order in the opening of the electronic gap, i.e. an unconventional Mott-Slater-type insulator. Finally, the sum-rules analysis ${ }^{46,47}$ indicates that the net orbital magnetic moment is $m_{1} \sim 0.003 \mu_{\mathrm{B}} / \mathrm{Ir}$ and the spin magnetic moment $m_{\mathrm{s}} \sim 0.007 \mu_{\mathrm{B}} / \mathrm{Ir}\left(0.005 \mu_{\mathrm{B}} / \mathrm{Ir}\right)$ if $T_{z}=0\left(14\left\langle T_{z}\right\rangle /\left\langle 4 S_{z}\right\rangle=\right.$ $\left.0.64^{45}\right)$ is assumed. The orbital to spin moment ratio is a factor $\sim 4$ smaller than the $m_{\mathrm{l}} / m_{\mathrm{s}}=2$ proposed for a $J_{\text {eff }}=1 / 2$ state, indicating a deviation from the pure $J_{\text {eff }}=1 / 2$ state.

\section{Conclusions}

The relationships between the structure of $\mathrm{IrO}_{2}$ thin films and their electrical and magnetic properties have been studied. The SOC in $\mathrm{IrO}_{2}$ thin films is very large and robust, being mostly independent of the structural details. A small ( 1-10\%) but reproducible increment of the SOC is observed for the thinnest samples. Regarding the electrical characterization, upon reducing the $\mathrm{IrO}_{2}$ film thickness, the response evolves from a metallic ground state to a strongly localized behavior as the RT sheet resistance approaches $\mathrm{h} / \mathrm{e}^{2} \approx 25 \mathrm{k} \Omega$ for $\sim 1.5 \mathrm{~nm}$. At around 2-3 nm-thickness, the films show upturns in resistivity at low temperatures. Upon further reducing the thickness of the deposited layer to $\sim 1.5 \mathrm{~nm}$, a small band gap is opened suggesting an enhanced role of electron correlations $(U / W)$. As for the magnetic properties, the temperature magnetization curves suggest the presence of magnetic ordering (canted AFM) at low temperatures in the thinnest samples. The XMCD measurements also support this hypothesis. In addition, it is found that the magnetic transition occurs at the same temperature at which the resistivity shows a strong increase, pointing to an interplay between magnetism and electrical behavior. Overall, our results are consistent with a combined effect of electron correlations and magnetic order as the mechanisms responsible for the thickness-dependent MIT found in $\mathrm{IrO}_{2}$ thin films (Mott-Slater-like insulator). This research highlights ultrathin $\mathrm{IrO}_{2}$ films as a novel platform for tuning the electrical and magnetic response of this material. Finally, it is worth noticing the potential relevance of combining the tunability in resistivity here shown with other interesting features of $\mathrm{IrO}_{2}$, namely, its large spin Hall effect (SHE), ${ }^{48}$ its nonsymmorphic crystalline symmetry that allows the switching of the charge carrier type ${ }^{29}$ and its easy growth relative to other iridates.

\section{Conflicts of interest}

There are no conflicts to declare.

\section{Acknowledgements}

This work was partially supported by the Spanish MINECO projects MAT2014-54425-R (MINECO/FEDER, UE), MAT201782970-C2-R (AEI/FEDER, UE), MAT2017-83468-R (AEI/FEDER, UE), MAT2017-87134-C02-01-R (AEI/FEDER, UE) and MAT201787134-C02-02-R (AEI/FEDER, UE), the Spanish MICINN project PID2020-115159GB-I00 / AEI / 10.13039/501100011033 and by the Aragon Regional Government (Projects No. E12-20R and E28-20R). E. A-E acknowledges the Spanish MINECO and the European Social Fund for an FPI (Formación de Personal Investigador, 2015) grant. R. B. acknowledges funding support from the European Union's Horizon 2020 research and innovation program under the Marie Skłodowska-Curie grant agreement no. 665919. This research used resources of the APS, a U. S. Department of Energy (DOE) Office of Science User Facility operated for the DOE Office of Science by Argonne National Laboratory under Contract No. DE-AC02-06CH11357. We acknowledge Diamond Light Source for time on I20-Scanning under Proposal SP-17266. The research leading to this result has been supported by the project CALIPSOplus under the Grant Agreement 730872 from the EU Framework Programme for Research and Innovation Horizon 2020. The authors acknowledge funding from the project Quantox of QuantERA ERA-NET Cofund of Quantum Technologies (Grant Agreement No. 731473) implemented within the European Union's Horizon 2020 Programme. The authors would like to acknowledge the use of Servicio General de Apoyo a la InvestigaciónSAI, Universidad de Zaragoza. The authors would like to acknowledge the use of the diffractometers at the Instituto de Nanociencia de Aragón and C. Munuera for AFM images.

\section{References}

1 D. Haskel, G. Fabbris, M. Zhernenkov, P. P. Kong, C. Q. Jin, G. Cao and M. van Veenendaal, Phys. Rev. Lett., 2012, 109, 027204.

2 G. Cao and P. Schlottmann, Rep. Prog. Phys., 2018, 81, 042502.

3 J. G. Rau, E. K.-H. Lee and H.-Y. Kee, Annu. Rev. Condens. Matter Phys., 2016, 7, 195-221.

4 W. Witczak-Krempa, G. Chen, Y. B. Kim and L. Balents, Annu. Rev. Condens. Matter Phys., 2014, 5, 57-82.

5 C. R. Serrao, J. Liu, J. T. Heron, G. Singh-Bhalla, A. Yadav, S. J. Suresha, R. J. Paull, D. Yi, J.-H. Chu, M. Trassin, A. Vishwanath, E. Arenholz, C. Frontera, J. Železný, T. Jungwirth, X. Marti and R. Ramesh, Phys. Rev. B: Condens. Matter Mater. Phys., 2013, 87, 085121.

6 J. Nichols, J. Terzic, E. G. Bittle, O. B. Korneta, L. E. D. Long, J. W. Brill, G. Cao and S. A. Seo, Appl. Phys. Lett., 2013, 102, 141908.

7 A. Lupascu, J. P. Clancy, H. Gretarsson, Z. Nie, J. Nichols, J. Terzic, G. Cao, S. S. A. Seo, Z. Islam, M. H. Upton, J. Kim, D. Casa, T. Gog, A. H. Said, V. M. Katukuri, H. Stoll, L. Hozoi, J. van den Brink and Y.-J. Kim, Phys. Rev. Lett., 2014, 112, 147201. 
8 D. J. Groenendijk, C. Autieri, J. Girovsky, M. C. MartinezVelarte, N. Manca, G. Mattoni, A. M. R. V. L. Monteiro, N. Gauquelin, J. Verbeeck, A. F. Otte, M. Gabay, S. Picozzi and A. D. Caviglia, Phys. Rev. Lett., 2017, 119, 256403.

9 J. Matsuno, K. Ihara, S. Yamamura, H. Wadati, K. Ishii, V. V. Shankar, H.-Y. Kee and H. Takagi, Phys. Rev. Lett., 2015, 114, 247209.

10 F.-X. Wu, J. Zhou, L. Y. Zhang, Y. B. Chen, S.-T. Zhang, Z.-B. Gu, S.-H. Yao and Y.-F. Chen, J. Phys.: Condens. Matter, 2013, 5, 125604.

11 J. H. Gruenewald, J. Nichols, J. Terzic, G. Cao, J. W. Brill and S. S. Seo, J. Mater. Res., 2014, 29, 2491-2496.

12 Y. F. Nie, P. D. C. King, C. H. Kim, M. Uchida, H. I. Wei, B. D. Faeth, J. P. Ruf, J. P. C. Ruff, L. Xie and X. Pan, Phys. Rev. Lett., 2015, 114, 016401.

13 A. Biswas, K.-S. Kim and Y. H. Jeong, J. Appl. Phys., 2014, 116, 213704.

14 J. Liu, J.-H. Chu, C. R. Serrao, D. Yi, J. Koralek, C. Nelson, C. Frontera, D. Kriegner, L. Horak, E. Arenholz, J. Orenstein, A. Vishwanath, X. Marti and R. Ramesh, 2013, arXiv:1305.1732v1 [cond-mat.str-el].

15 Q. Cui, J.-G. Cheng, W. Fan, A. E. Taylor, S. Calder, M. A. McGuire, J.-Q. Yan, D. Meyers, X. Li, Y. Q. Cai, Y. Y. Jiao, Y. Choi, D. Haskel, H. Gotou, Y. Uwatoko, J. Chakhalian, A. D. Christianson, S. Yunoki, J. B. Goodenough and J.-S. Zhou, Phys. Rev. Lett., 2016, 117, 176603.

16 C. Lu, A. Quindeau, H. Deniz, D. Preziosi, D. Hesse and M. Alexe, Appl. Phys. Lett., 2014, 105, 082407.

17 J. Cheng, J. Zhou, J. B. Goodenough, K. Matsubayashi and Y. Uwatoko, JPS Conf. Proc., 2014, 3, 013014.

18 S. K. Panda, S. Bhowal, A. Delin, O. Eriksson and I. Dasgupta, Phys. Rev. B: Condens. Matter Mater. Phys, 2014, 89, 155102.

19 J. M. Kahk, C. G. Poll, F. E. Oropeza, J. M. Ablett, D. CÃolin, J. Rueff, S. Agrestini, Y. Utsumi, K. D. Tsuei and Y. F. Liao, Phys. Rev. Lett., 2014, 112, 117601.

20 X. Ming, K. Yamauchi, T. Oguchi and S. Picozzi, 2017, arXiv:1702.04408 [cond-mat.str-el], 89.

21 M. Varela, W. Grogger, D. Arias, Z. Sefrioui, C. León, C. Ballesteros, K. M. Krishnan and J. Santamaría, Phys. Rev. Lett., 2001, 86, 5156-5159.

22 G. Sanchez-Santolino, J. Tornos, D. Hernandez-Martin, J. I. Beltran, C. Munuera, M. Cabero, A. Perez-Munoz, J. Ricote, F. Mompean, M. Garcia-Hernandez, Z. Sefrioui, C. Leon, S. J. Pennycook, M. C. Munoz, M. Varela and J. Santamaria, Nat. Nanotechnol., 2017, 12, 655-662.

23 M. Cabero, K. Nagy, F. Gallego, A. Sander, M. Rio, F. A. Cuellar, J. Tornos, D. Hernandez-Martin, N. M. Nemes, F. Mompean, M. Garcia-Hernandez, A. Rivera-Calzada, Z. Sefrioui, N. Reyren, T. Feher, M. Varela, C. Leon and J. Santamaria, APL Mater., 2017, 5, 096104.

24 L. J. van der Pauw, Philips Res. Rep., 1958, 13, 1.

25 S. Diaz-Moreno, M. Amboage, M. Basham, R. Boada, N. E. Bricknell, G. Cibin, T. M. Cobb, J. Filik, A. Freeman,
K. G. D. Gianolio, S. Hayama, K. Ignatyev, L. Keenan, I. Mikulska, J. F. W. Mosselmans, J. J. Mudd and S. A. Parry, J. Synchrotron Radiat., 2018, 25, 998.

26 S. Diaz-Moreno, S. Hayama, M. Amboage, A. Freeman, J. Sutter and G. Duller, J. Phys.: Conf. Ser., 2009, 190, 012038.

27 S. Hayama, G. Duller, J. P. Sutter, M. Amboage, R. Boada, A. Freeman, L. Keenan, B. Nutter, L. Cahill, P. Leicester, B. Kemp, N. Rubies and S. Diaz-Moreno, J. Synchrotron Radiat., 2018, 25, 1556.

28 E. Arias-Egido, M. A. Laguna-Marco, C. Piquer, R. Boada and S. Diaz-Moreno, Adv. Funct. Mater., 2019, 29, 1806754.

29 M. Uchida, W. Sano, K. S. Takahashi, T. Koretsune, Y. Kozuka, R. Arita, Y. Tokura and M. Kawasaki, Phys. Rev. $B, 2015,91,241119$.

30 A. Bose, J. N. Nelson, X. S. Zhang, P. Jadaun, R. Jain, D. G. Schlom, D. C. Ralph, D. A. Muller, K. M. Shen and R. A. Buhrman, ACS Appl. Mater. Interfaces, 2020, 12, 55411-55416.

31 W. J. Kim, S. Y. Kim, C. H. Kim, C. H. Sohn, O. B. Korneta, S. C. Chae and T. W. Noh, Phys. Rev. B, 2016, 93, 045104.

32 L. Wang, L. Chang, X. Yin, A. Rusydi, L. You, Y. Zhou, L. Fang and J. Wang, J. Phys.: Condens. Matter, 2017, 29, 025002 .

33 X. Shen, X. Qiu, D. Su, S. Zhou, A. Li and d D. Wu, J. Appl. Phys., 2015, 117, 015307.

34 R. Scherwitzl, S. Gariglio, M. Gabay, P. Zubko and M. G. M. Triscone, Phys. Rev. Lett., 2011, 106, 246403.

35 J. K. Kawasaki, D. Baek, H. Paik, H. P. Nair, L. F. Kourkoutis, D. G. Schlom and K. M. Shen, Phys. Rev. Mater., 2018, 2, 054206.

36 L. Hao, D. Meyers, C. Frederick, G. Fabbris, J. Yang, N. Traynor, L. Horak, D. Kriegner, Y. Choi, J.-W. Kim, D. Haskel, P. J. Ryan, M. P. M. Dean and J. Liu, Phys. Rev. Lett., 2017, 119, 027204.

37 D. C. Licciardello and D. J. Thouless, Phys. Rev. Lett., 1975, 35, 1475-1478.

38 N. F. Mott, Metal Insulator Transitions, Taylor and Francis, London, 1990.

39 N. F. Mott and E. A. Davies, Electron Processes in NonCrystalline Materials, Clarendon, Oxford, 1979.

40 A. L. Efros and B. I. Shklovskii, J. Phys. C: Solid State Phys., 1975, 8, L49-L51.

41 J. K. Kawasaki, C. H. Kim, J. N. Nelson, S. Crisp, C. J. Zollner, E. Biegenwald, J. T. Heron, C. J. Fennie, D. G. Schlom and K. M. Shen, Phys. Rev. Lett., 2018, 121, 176802.

42 F. Ye, S. Chi, B. C. Chakoumakos, J. A. Fernandez-Baca, T. Qi and G. Cao, Phys. Rev. B: Condens. Matter Mater. Phys., 2013, 87, 140406R.

43 D. Haskel, G. Fabbris, M. Zhernenkov, P. Kong, C. Jin, G. Cao and M. van Veenendaal, Phys. Rev. Lett., 2012, 109, 027204.

44 M. A. Laguna-Marco, P. Kayser, J. Alonso, M. MartinezLope, M. van Veenendaal, Y. Choi and D. Haskel, Phys. Rev. B., 2015, 91, 214433. 
45 M. A. Laguna-Marco, D. Haskel, N. Souza-Neto, J. C. Lang, V. V. Krishnamurthy, S. Chikara, G. Cao and M. van Veenendaal, Phys. Rev. Lett., 2010, 105, 216407.

46 B. T. Thole, P. Carra, F. Sette and G. van der Laan, Phys. Rev. Lett., 1992, 68, 1943.
47 P. Carra, B. T. Thole, M. Altarelli and X. Wang, Phys. Rev. Lett., 1993, 70, 694.

48 K. Fujiwara, Y. Fukuma, J. Matsuno, H. Idzuchi, Y. Niimi, Y. Otani and H. Takagi, Nat. Commun., 2013, 4, 2893. 